\title{
MEETING OF THE ASSOCIATION FOR SYMBOLIC LOGIC
}

A meeting of the Association for Symbolic Logic was held in Stamford Hall, University of Leicester, Leicester, England from 7-11 September 1965 as the last three days of a Summer School in Mathematical Logic.

One hour invited adresses were given by Prof. J. C. E. Dekker, Prof. A. Nerode, Prof. G. E. Sacks, Prof. Hartley Rogers, Jr., Prof. Dr. G. Hasenjaeger, Dr. R. B. Jensen. Dr. Haim Gaifman and Prof. Simon Kochen. In addition seventeen twenty minute papers were given. The last nine abstracts below are of papers presented by title.

J. N. Crossley

P. H. G. Aczel. Paths in Kleene's O.

A path in $O$ is a linearly ordered subset of $O$, closed under predecessors (with respect to $\left.\leqq_{0}\right)$. The r.e. paths in $O$ are just those of the form $\left\{x: x<_{0}\right.$ a $\}$ for some a $\varepsilon O$.

A well-ordering $\mathfrak{A}$ is semi-regressive if $\{y:\langle y, x\rangle \in \mathfrak{A} \& \mathrm{y} \neq \mathrm{x}\}$ is uniformly r.e. in $\mathbf{x}$, for $\mathbf{x}$ in the field of $\mathfrak{A}$. Clearly every r.e. well-ordering is semi-regressive.

Theorem 1. Let $|\mathrm{P}|=\omega^{\lambda}$

i) $\mathrm{P}$ is the co-ordinal of a path in $O$, if and only if $\mathrm{P}=\mathrm{W} . \mathrm{A}$ for some co-ordinal A of a semi-regressive well-ordering.

ii) $\mathrm{P}$ is the co-ordinal of a r.e. path in $O$, if and only if $\mathrm{P}=\mathrm{W}$.A for some co-ordinal A of a r.e. well-ordering.

Theorem 2. i) Every non-r.e. path in $O$ has order type of the form

$$
\omega^{2} \lambda, \quad\left(0<\lambda \leqq \omega_{1}\right) \text {. }
$$

ii) For each $\lambda$ such that $0<\lambda \leqq \omega_{1}$, there are $2^{\aleph_{0}}$ paths in $O$ of order type $\omega^{2} \lambda$.

Theorem 3. i) Every regressive function enumerates (in increasing order) a semiregressive well-ordering of order type $\omega$.

ii) The field of a non-r.e. semi-regressive well-ordering of order type $\omega$ is immune. (Received 30 July, 1965.)

G. Beaman. Decidability and recursiveness.

In the accepted terminology a predicate $\mathrm{p}$ is called effectively decidable if there exists a uniform method of deciding px, for all $\mathrm{x}$. Since uniformity of method is perhaps not of exclusive importance let us consider decidability which is not necessarily uniform.

A statement $\mathrm{S}$ is decidable if and only if there exists an adequate correct argument showing whether $\mathrm{S}$ is true or false. We do not know how to characterise adequate correct arguments precisely but may say that a contradictory argument is not correct and a guess is not adequate.

A predicate $\mathrm{p}$ is decidable if and only if for all $\mathrm{x} \mathrm{px}$ is decidable.

Church's thesis is that every effectively decidable predicate is general recursive. It may be asked whether every decidable predicate is general recursive. In this connection we prove a

Theorem. If every decidable predicate is general recursive then there exists a statement which is not decidable.

Suppose that every decidable predicate is general recursive. Then since $\forall y \overline{T_{1}(x, x, y)}$ (reference 1) is not general recursive it is not decidable. Hence there exists $x$ such that $\forall y \overline{T_{1}(x, x, y)}$ is not decidable. (Note that an $\mathrm{x}$ with this property cannot be known.)

The undecidability of such a statement is absolute in that our definition of decidability is as liberal as it can be.

[1] Kleene: Recursive predicates and quantifiers. (Trans. A.M.S. 53 (1943))

[2] Kalmár: An argument against the plausibility of Church's thesis. (Heyting: Constructivity in Mathematics) 
[3] Mendelson: On some recent criticism of Church's thesis. (Notre Dame J. Formal Logic 4 (1963)) (Received 5 July, 1965.)

J. P. Cleave. Bounded ultraproducts.

1) $H A$ ultrafilters

$\mathrm{N}$ denotes the set of natural numbers.

Let $\Omega, \Gamma \subseteq \mathrm{N}$.

$$
\Omega=\omega_{\mathbf{x}} \leftrightarrow \Omega_{\mathrm{T}} \underset{\mathrm{H}}{\mathrm{H}}(\mathrm{x})_{1} \text { with Gödel number }(\mathrm{x})_{\mathbf{0}} .
$$

Let $\alpha, \beta \varepsilon 0 . \Omega$ is a $H A$ ultrafilter of $\operatorname{order}(\alpha, \beta)$ in $\Gamma$ if and only if (i) $\Omega \leq \underset{\mathrm{T}}{\leq} \mathrm{H}_{\beta}^{\Gamma}$, (ii) $(\mathrm{x})_{\Omega}\left((\mathrm{x})_{1}<\alpha\right)$, (iii) $\left\{\omega_{\mathrm{x}} \mid \mathrm{x} \varepsilon \Omega\right\}$ is an ultrafilter wrt the Boolean algebra of sets $\leq \mathrm{H}_{\mathbf{y}}^{\Gamma}$ for some $\mathrm{y}<\alpha$.

2) Bounded ultraproducts

$\left\{U_{1}\right\}_{i \varepsilon N}$ is a family of denumerable systems in a similarity class $K$. Let $\Gamma$ be a set in which the $\mathrm{U}_{1}$ are uniformly recursive. Let $\alpha<\beta \varepsilon 0$ and let $\mathrm{D}$ be an ultrafilter of order $(\alpha, \beta)$ in $\Gamma$

$$
\Pi^{\alpha}\left|U_{i}\right|=\left\{f \mid(i)_{N}\left(f(i) \varepsilon\left|U_{i}\right|\right) \& f \underset{T}{\leq} H_{y}^{\Gamma} \text { for some } y<\alpha\right\} .
$$

D determines an equivalence relation on $\Pi^{\alpha}\left|U_{\mathbf{i}}\right| . \Pi^{\beta}\left|U_{\mathbf{i}}\right| / D$ is the domain of the bounded ultraproduct $\Pi^{\beta} \mathrm{U}_{1} / \mathrm{D}$. Product relations are defined in the usual manner (Frayne, Morel, Scot Fund. Math. LI (1962)).

Theorem 1 . If $\alpha$ is a limit ordinal and $\Phi \varepsilon \mathrm{L}(\mathrm{K})$, then

(i) $\frac{\Pi^{\alpha} \mathrm{U}_{1}}{\mathrm{D}} \vDash \Phi \leftrightarrow\left\{\mathrm{i}\left|\mathrm{U}_{\mathbf{i}}\right| \vDash \Phi\right\} \varepsilon \mathrm{I}$

(ii) $\frac{\Pi^{\alpha} U_{1}}{\mathrm{D}} \leq \mathrm{H}_{\beta}^{\Gamma}$

$\frac{\Pi^{\alpha} U_{1}}{D}$ is denumerable; the axiom of choice is not required in Th. 1.

3) Elementary Equivalence

If $U_{i}=U$ for all $i \varepsilon N$ write $\frac{U^{N}, \alpha}{D}$ instead fof $\frac{\Pi^{\alpha} U_{i}}{D}$. We have the following version of Keisler's characterisation of elementary equivalence (Indag. Math. 23 (1961)).

Theorem 2. Let $\mathrm{U}, \mathrm{B}$ be denumerable systems each recursive in $\Gamma$.

If $\mathrm{U} \equiv \mathrm{B}$, there exist ultrafilters $\mathrm{D}, \mathrm{E}$ of orders $\left(\omega^{2}, \omega^{2}+\omega\right)$ and $\left(\omega^{2}, \omega^{2}+2 \omega\right)$ resp., in $\Gamma$ such that

$$
\frac{\mathrm{U}}{\mathrm{D}}^{\mathrm{N}, \omega^{2}} \cong{\frac{\mathrm{B}}{\mathrm{E}, \omega^{2}}}
$$

The continuum hypothesis is not required for this result. (Received I June, I965.)

J. N. Crossley. Infinite sums of co-ordinals.

Let $R$ be a fixed recursive dense linear ordering of the non-negative integers with first element but no last element. Let $L$ be the category of all $1-1$ partial recursive maps which preserve the ordering of $R$. Let $\mathfrak{A}, \mathfrak{B}$ be r.e. subsets of $R$ and let $f \varepsilon L$. $\mathrm{F}$ is said to be an effective functor on $\mathrm{L}$ if i) $\mathrm{F}$ is an effective operation and ii) $\mathrm{F}$ is a functor from $\mathbf{L}$ to $\mathbf{L}$.

Theorem. If $\mathrm{F}$ is an effective functor then

$$
\Sigma F^{n}(\mathrm{~A})=\operatorname{CRT}\left(\Sigma F^{n}(\mathfrak{A})\right)
$$

can be well-defined for $\mathfrak{A} \varepsilon \mathrm{A}$. 
Corollary. If $\mathrm{F}$ is an effective functor satisfying certain uniformity conditions (where $\mathrm{F}(\mathrm{A})=\mathrm{CRT}(\mathrm{F}(\mathfrak{M}))$ ) then $\lim \mathrm{F}^{\mathrm{n}}(\mathrm{A})$ can be well defined.

The above correspond to the case of successor being recursively representable on a well-ordering in the sense of abstract XXVIII 308. The method extends to cases a) $-f)$ of the theorem given there.

Note. S. Feferman has pointed out that the definition in XXVIII 308 should be amended to: $\varphi$ is recursively representable on $\mathfrak{A}$ if there is a partial recursive function $f$ such that if $a_{i} \varepsilon C^{\prime} \mathfrak{A}$ then

(i) $\quad \varphi\left(\left|\mathrm{a}_{1}\right|, \ldots,\left|\mathrm{a}_{\mathbf{n}}\right|\right)<|\mathfrak{Q}| \leftrightarrow \mathrm{f}\left(\mathrm{a}_{1}, \ldots, \mathrm{a}_{n}\right)-1 \varepsilon \mathrm{C}^{\prime} \mathfrak{A}$ and (ii) $\left|\mathrm{f}\left(\mathrm{a}_{1}, \ldots, \mathrm{a}_{\mathrm{n}}\right)-1\right|=$ $\varphi\left(\left|\mathrm{a}_{1}\right|, \ldots,\left|\mathrm{a}_{\mathbf{n}}\right|\right)$ where $\mathbf{f}(\mathrm{x})-1=\mu_{\mathrm{y}}\{\mathrm{f}(\mathrm{x})=\mathrm{y}+1\}$. Theorem part e) must also be amended by stipulating $\alpha_{\mathbf{i}} \leqq \varphi_{\mathbf{j}}\left(\alpha_{1}, \ldots, \alpha_{r}\right)$ in the hypothesis. (Received $\left.20 \mathrm{July}, 1965.\right)$

A. LUIS DARzins. Logical truth matrices, probability calculus and continuity.

1. Some problems in the derivation of probable truth values of a) propositions, where these truth values are derived from the truth value of the logical operation (or sign) in i. the two truth value case, and ii. the many valued (Post) logic case; b) the first, and second clauses of propositions; c) propositional functions.

2. The determinacy of equi-probably true propositions derived from the truth value of logical operations (signs).

3. Continuity and discontinuity of probably true propositional functions. (Received I5 July, I965.)

J. C. E. Dekker. Regressive isols.

A survey of the results concerning regressive isols obtained by the author, J. Barback, E. Ellentuck, D. C. Ferguson, M. J. Hassett and F. J. Sansone. (Received 28 July, 1965.$)$

J. DeRRICK and F. R. DRAKE. Independence of the axiom of choice at higher cardinalities.

The following theorem is proved using techniques of Cohen and Feferman.

Let $\mathrm{M}$ be a model of set theory $\Sigma(Z F$ or VNB) together with the axiom of choice, for which $2^{\boldsymbol{N}_{\alpha}}=\boldsymbol{N}_{\mathrm{G}^{*} \alpha}$ [See W. Easton, Powers of regular cardinals, Ph.D. Thesis, Princeton 1964, for possible values of G].

Let $\kappa$ be any infinite regular cardinal of $\mathrm{M}$ for which

$$
\boldsymbol{\psi}_{x}<\kappa \text { implies } 2^{\boldsymbol{N}_{\alpha}} \leq \kappa
$$

(i.e. the cardinality of $S_{\kappa}(\kappa)$ is $\kappa$, where $S_{\kappa}(\kappa)$ is the set of all subsets of $\kappa$ of cardinality $<\kappa$ ).

Then there is a model $\mathrm{N}$ of $\mathrm{\Sigma}$ which is an extension of $\mathrm{M}$ in which

(1) the axiom of choice holds for sets of cardinality $<\kappa$ (so if $h$ is a function mapping $\mathrm{x}$ one-one onto an ordinal $\alpha<\kappa$, there is a function $\mathrm{g}$ on $\alpha$ such that $\mathrm{g}(\alpha) \varepsilon \mathrm{h}^{-1}(\alpha)$ if $\left.\mathrm{h}^{-1}(\alpha) \neq \varnothing\right)$,

(2) $2^{\boldsymbol{N}_{\alpha}}=\boldsymbol{\kappa}_{\mathbf{G}^{\prime} \alpha}$ if $\boldsymbol{\kappa}_{\alpha}<\kappa$,

(3) the prime ideal theorem fails for $2^{\kappa}$ : in particular there is no prime ideal containing $S_{\kappa}(\kappa)$.

So that in $\mathrm{N}$ the axiom of choice fails first at cardinality $к$. (Received $2 I J u l y, r 965$.

J. DERRICK and F. R. DRAKE. Independence of the axiom of choice from a variant of the generalized continuum hypothesis.

For any model $\mathrm{K}$ of the set theory $\Sigma$, let $\boldsymbol{N ( K )}$ be Hartogs' aleph function, i.e. for any set $\mathrm{x}$ of $\mathrm{K}, \boldsymbol{N}(\mathrm{K})(\mathrm{x})$ is the least cardinal of $\mathrm{K}$ which is not similar in $\mathrm{K}$ to a subset of $\mathrm{x}$. 
Then in the model $\mathrm{N}$ of the preceding abstract, the following also holds:

$$
\boldsymbol{\aleph}^{(\mathrm{N})}\left(2 \boldsymbol{N}_{\alpha}\right)=\boldsymbol{\aleph}_{\left(\mathrm{G}^{\prime} \alpha\right)+1} \quad \text { for all } \alpha .
$$

(This is proved using methods derived from Solovay.)

Note that in $\Sigma$, (4) with the axiom of choice would imply

$$
2^{\boldsymbol{N}_{\alpha}}=\boldsymbol{K}_{\mathbf{G}}{ }^{\prime} \alpha \quad \text { for all } \alpha \text {, }
$$

and we have shown that the axiom of choice is independent of $\Sigma+(4)$. Thus if

$$
\mathrm{G}^{\prime} \alpha=\alpha+1 \quad \text { for all } \alpha,
$$

(4) becomes a weaker variant of the generalized continuum hypothesis:

$$
\boldsymbol{N}\left(2^{\left.\boldsymbol{N}_{\alpha}\right)}=\boldsymbol{\aleph}_{\alpha+2}\right.
$$

and the axiom of choice cannot be deduced from this in $\Sigma$. (Received $2 I$ July, 1965. )

WALTER FELSCHER. Theorems on general algebras which hold without the axiom of choice.

A type is a sequence $T=\left(K_{i}\right)_{i} \epsilon_{I}$ of sets. An algebra of type $T$ is a pair $A=\left\langle A_{0}\right.$, $\left(f_{1}\right)_{i \in}>$ such that $f_{i}: A K_{0}^{1} \rightarrow A_{0}$ for $i \varepsilon I$. A type or an algebra is finitary if every $K_{i}$ is finite; it is of wo-type if every $\mathrm{K}_{\mathrm{i}}$ is an ordinal. KERKHOFF (Math. Ann. 158 (1965), 109-112) has shown without the axiom of choice (AC): for any type T and any set $\mathrm{X}$ there exists an algebra of type $\mathrm{T}$, absolutely freely generated by $\mathrm{X}$. Therefore, if a class $\mathrm{C}$ of finitary algebras is closed under subdirect products and isomorphisms, there exists without (AC) for any set $\mathrm{X}$, an algebra in $\mathrm{C}, \mathrm{C}$-freely generated by $\mathrm{X}$. For finitary algebras one can prove without (AC): (1) A class $\mathrm{C}$ of algebras is equationally definable iff it is primitive, i.e. closed under products, subalgebras and homomorphic images: $\mathrm{P}(\mathrm{C}) \subseteq \mathrm{C}, \mathrm{S}(\mathrm{C}) \subseteq \mathrm{C}, \mathrm{H}(\mathrm{C}) \subseteq \mathrm{C}$ (BIRKHOFF). (2) For any class $\mathrm{C}$ the class $\operatorname{HSP}(C)$ is the smallest primitive class containing $C$. (3) if $C$ is primitive and A is functionally free in $\mathrm{C}$ then $\mathrm{C}=\operatorname{HSP}(\{\mathrm{A}\})$ (TARSKI). In particular, without (AC) any Boolean algebra is a homomorphic image of a field of sets. Further, for finitary algebras the compactness theorem holds without (AC): if $\mathrm{M}$ is a set of equations such that every finite subset of $M$ possesses a model with more than one element, then $M$ possesses a model with more than one element. (Received I5 July, I965.)

Jens Erik Fenstad. A limit theorem in polyadic probabilities.

Let $\mathrm{A}$ be a denumerable, locally finite polyadic algebra with equality. Denote by $S$ $\left(S_{0}\right)$ the space of all (finite) equality models of A. Let $t_{n}, n=1,2, \ldots$, be the elements which express that there are exactly $n$ individuals. As a notational device let A contain elements $v_{n}, n=1,2, \ldots$, denoting individuals, i.e.

$$
\exists(1)\left[v_{n} \wedge \forall(2)\left[\left(S\left(\frac{1}{2}\right) v_{n}\right)^{\prime} \vee E(1,2)\right]\right]=1
$$

is valid in $A$ (assuming that support of $v_{\mathbf{n}}=\{1\}$ ). Assume further that A satisfies the inequalities

$$
t_{n} \leq \wedge \forall(1)\left[v_{i}^{\prime} \vee v_{j}^{\prime}\right], \quad 1 \leqq i, j \leqq n, \quad i \neq j,
$$

which means that if $M \varepsilon S_{0}$ has $n$ individuals, each of them is named by some $v_{\mathbf{i}}$.

Let $c$ be a probability on $\mathrm{A}$, i.e. $0 \leqq c(\mathrm{p}) \leqq 1, c(0)=0, c(1)=1$ and $c(\mathrm{p})+c(\mathrm{q})=$ $c(\mathrm{p} \vee \mathrm{q})+c(\mathrm{p} \wedge \mathrm{q})$. If $\mathrm{B}=\left\{\mathrm{q}_{\mathrm{n}}\right\}$ is any set of sentences of $\mathrm{A}$, define $c(\mathrm{~B})=$ $\lim c\left(\mathrm{q}_{1} \wedge \ldots \wedge \mathrm{q}_{\mathrm{m}}\right)$. We shall suppose that $c$ describes individuals in models as equiprobable and that no extended axiom of infinity shall receive positive probability, i.e. $c$ satisfies $(\mathrm{I}): c\left(\mathrm{v}_{\mathrm{1}} \wedge \mathrm{q}\right)=c\left(\mathrm{v}_{\mathrm{j}} \wedge \mathrm{q}\right)$, for all sentences $\mathrm{q}$, and (II): If $c(\mathrm{~B})>0$, $B$ a set of sentences, then for some $n, q_{1} \wedge \ldots \wedge q_{m} \wedge t_{n} \neq 0$ for all $q_{1}, \ldots, q_{m} \varepsilon B$. 
If $p \varepsilon A$ is of support one, let $\operatorname{fr}(p, M), M \varepsilon S_{0}$, denote the relative frequency of (the property) $\mathrm{p}$ in $\mathrm{M}$.

Theorem. $\frac{1}{\mathrm{n}} \Sigma \mathrm{fr}\left(\mathrm{p}, \mathrm{M}_{\mathbf{i}}\right)$ converges almost certainly to $c(\mathrm{p})$. The proof uses the following facts: (i) every $c$ on A can be represented in the form $c(\mathrm{p})=\int \mu_{\mathrm{M}}(\mathrm{p}[\mathrm{M}]) \mathrm{d} \lambda(\mathrm{M})$, where $\lambda$ is a $\sigma$-additive probability measure on $\mathrm{S}, \mu_{\mathrm{M}}$ a probability on the sets $\mathrm{p}[\mathrm{M}]=\left\{\mathrm{x} \in \mathrm{X}_{\mathrm{M}}^{\mathrm{I}}: \mathrm{p}_{\mathrm{M}}(\mathrm{x})=1\right\}$; (ii) $\mu_{\mathrm{M}}(\mathrm{p}[\mathrm{M}])=\mathrm{fr}(\mathrm{p}, \mathrm{M})$ if $\mathrm{M} \varepsilon \mathrm{S}_{0}$; and (iii) $\lambda\left(\mathrm{S}_{0}\right)=1$. The proof then consists in an application of the strong law of large numbers. (Received 23 July, 1965.)

R. B. JENSEN. Independence of the axiom of dependent choices from the countable axiom of choice.

If $\mathrm{ZF}$ is consistent, then it remains consistent after adjoining the following axioms:

(1) The countable axiom of choice,

(2) There is a relation $\mathrm{R}$ such that for every real $x$ a real $y$ exists such that: Rxy but there is no sequence $\left\{x_{i}: i<\omega\right\}$ such that: $\mathrm{R} x_{i} x_{i+1}$ for all $i$. (Received II September, 1965.$)$

CAROL KARP. Primitive recursive set functions: a formulation with applications to infinitary formal systems.

Call a set function $F\left(x_{1}, \ldots, x_{n}\right)$ finitarily primitive recursive (f.p.r.) if it arises by composition, set recursion, and the set formation schema $F\left(x_{1}, \ldots, x_{n}\right)=$ $\left\{G\left(z, x_{1}, \ldots, x_{n}\right) \mid z \varepsilon x_{1}\right\}$, from a set of initial functions consisting of the projections, the pairing function, union, difference, $\omega$ and 0 . Gödel's $F$-function generating the constructible sets is an example of an f.p.r.function (f.p.r.f.).

Let $\Omega$ be a regular, non-denumerable cardinal and let $T_{\Omega}$ be the set of all sets hereditarily of power less than $\Omega$. Then $T_{\Omega}$ is closed under f.p.r.f.'s and the Löwenheim-Skolem Theorem for $(\Omega, \omega)$-languages implies that for $x_{1}, \ldots, x_{n} \varepsilon T_{\Omega}$, if $\mathrm{R}$ is a f.p.r. relation and $(\exists y) R\left(x_{1}, \ldots, x_{n}, y\right)$ holds in $V$ then it holds in $T_{\Omega}$. Applying this to Gödel's $F$, we have yet another proof that $V=L$ implies the generalized continuum hypothesis. The $\Omega$-primitive recursive functions ( $\Omega$-p.r.f.'s) are restrictions to $T_{\Omega}$ of functions $F\left(x_{1}, \ldots, x_{n}\right)=G\left(x_{1}, \ldots, x_{n}, s_{1}, \ldots, s_{m}\right), s_{1}, \ldots, s_{m} \varepsilon T_{\Omega}$, $G$ an f.p.r.f. Proof predicates for the basic $\Omega$-formal systems as well as computation predicates for the $\Omega$-recursive functions of ordinals, are $\Omega$-p.r. The $\Omega$-p.r.f.'s can be represented in a simple $(\Omega, \omega)$-formal system with an $\Omega$-p.r. proof predicate. The enumeration theorem follows for $\Omega$-recursively enumerable $\left(\Sigma_{1}-\Omega\right.$-p.r.) predicates.

For infinite cardinals $\kappa$, the $\kappa^{+}$-propositional formulas are like ordinary propositional formulas except that conjunctions and disjunctions of length $<\kappa^{+}$are admitted. Theorem 1. If $\kappa$ is a strong limit cardinal of cofinality $\omega$, then the $\kappa^{+}$-tautologies are $\kappa^{+}$-p.r. axiomatizable. Theorem 2. If $\kappa \exp \omega=\kappa$, then the $\kappa^{+}$-tautologies are not $\kappa^{+}$-p.r. axiomatizable. Thus assuming the generalized continuum hypothesis, the $\kappa^{+}$-tautologies are $\kappa^{+}$-p.r. axiomatizable iff $\operatorname{cf}(\kappa)=\omega$. The methods are sufficiently general that these theorems could be expected to hold for any reasonable notion of $\kappa^{+}$-recursiveness. (Received 20 July, I965.)

F. William Lawvere. Functorial semantics of elementary theories.

By an elementary theory we mean a small category $\mathrm{T}$ with the following structure: a) There are two distinguished objects $A, L$ and all objects are precisely the finite categorical products $A^{n} \times L^{k}$ b) For each object $X, L^{k} \times X$ is the $2^{k}$-fold coproduct (sum) of $\mathrm{X}$ with itself; e.g. $\mathrm{L}=1+1$. c) The $2^{\mathrm{k}}$-fold product of $\mathrm{X}$ with itself is the categorical exponential " $X$ to the power $L^{k}$ " [The more precise statement of a), b), c) is that there are given projections, injections, and evaluation maps satisfying 
usual universal mapping properties.] d) For every pair $\mathrm{f}: \mathrm{X} \rightarrow \mathrm{Y}, \varphi: \mathrm{X} \rightarrow \mathrm{L}$ in $\mathrm{T}$ there is $\exists_{\mathrm{f}}[\varphi]: \mathrm{Y} \rightarrow \mathrm{L}$ such that for every $\psi: \mathrm{Y} \rightarrow \mathrm{L}$ one has $\exists_{\mathrm{f}}[\varphi] \leq_{\mathrm{Y}} \psi$ iff $\varphi \leq \mathrm{x}$ f Here $\leq \mathbf{x}$ is the partial order of a canonical Boolean structure which the set of all maps $\mathrm{X} \rightarrow \mathrm{L}$ enjoys by virtue of a), b), c). Every "applied first-order 'theory' with equality" gives rise to an elementary theory in which (equivalence classes of) n-ary terms become maps $\mathrm{A}^{\mathrm{n}} \rightarrow \mathrm{A}$ and $\mathrm{n}$-ary formulas become maps $\mathrm{A}^{\mathrm{n}} \rightarrow \mathrm{L}$; the above conditions imply that all maps in $\mathrm{T}$ are in one way or another "tuples" of maps of these two special kinds. Other consequences include the existence of a map $A^{2} \rightarrow L$ playing the role of equality, and for every sentence $\varphi: 1 \rightarrow \mathrm{L}$ a corresponding binary function symbol $\mathrm{A}^{2} \rightarrow \mathrm{A}$ which in any model denotes one or other of the projections depending on the truth or falsity of $\varphi$ in the model. Every instance of $\exists_{f}[\varphi]$ is equal to a certain logical combination of special instances which correspond to the usual quantifiers (namely instances in which $f$ is a projection $A^{n+1} \rightarrow A^{n}$ ). A model is a functor $\mathrm{M}: \mathrm{T} \rightarrow \mathscr{S}$ to the category $\mathscr{P}$ of sets (Lawvere PNAS Dec. 1964) which preserves the additional structure (in particular (L)M is a two element set and $\left(\exists_{f}[\varphi]\right) \mathrm{M}$ is the indicator of the image under (f) $\mathrm{M}$ of the subset whose indicator is $(\varphi) \mathrm{M}$; the set (A) $\mathrm{M}$ is the universe of the model. Natural transformations between models are exactly elementary embeddings, yielding an "elementary category" $\mathscr{S}^{[\mathrm{T}]}$. Any morphism $\mathrm{T}^{\prime} \rightarrow \mathrm{T}$ of theories yields an "elementary functor" $\mathscr{S}[\mathrm{T}] \rightarrow$ $\left.\mathscr{P}^{[\mathrm{T}}\right]$ ] which preserves universes ("reducts" are special cases of elementary functors). Interpreting implicit definability in terms of natural transformations yields a functor "elementary structure" adjoint to the single large functor "elementary semantics" defined by the foregoing sentence. Given a first-order language-with-axioms $\mathscr{L}$, the associated theory T may be thought of as the "Sinn" of $\mathscr{L}$ and the category $\mathscr{S}[\mathrm{T}]$ as the "Bedeutung" of $\mathscr{L}$; a similar remark applies to the algebraic theories previously discussed (Lawvere, PNAS, Nov. 1963) and to a notion of "hypertheory" now being investigated. From this approach results a canonical procedure for embedding elementary categories and hypercategories as full subcategories of equationally definable categories of algebras. (Received 17 Aug., I965.)

TheOdORE A. LINDEN. Equivalences between Gödel's definitions of constructibility.

The definitions of constructibility given by Kurt Gödel in his paper on the consistency of the continuum hypothesis and in his later monograph are different. But they can be proved equivalent not only for the class of all constructible sets, but also at each $\omega_{\gamma}$, where $\omega_{\gamma}$ is the least ordinal of cardinality $\gamma$. That is, we always have $M_{\omega_{\gamma}}=\mathrm{F}\left(\omega_{\gamma}\right)$. The proof is by transfinite induction on the statement, "For $\alpha<\omega_{\gamma}$ there exists an $\alpha^{\prime}<\omega_{\gamma}$ such that $\mathrm{M}_{\alpha}=\mathrm{F}\left(\alpha^{\prime}\right)$." Take $\alpha_{1}<\omega_{\gamma}$, we show that for any set $\mathbf{A}$ which is first order definable over $\mathbf{F}\left(\alpha_{1}\right)$, there is an $\alpha_{2}<\omega_{\gamma}$ such that $\mathbf{A}=$ $F\left(\alpha_{2}\right)$. It then follows that there is an $\alpha_{3}<\omega_{y}$ such that $\mathfrak{F}\left(F\left(\alpha_{1}\right)\right)$, the class of everything first order definable over $F\left(\alpha_{1}\right)$, is a subset of $F\left(\alpha_{3}\right)$. Furthermore $\alpha_{3}$ can be chosen so that $\mathfrak{F}\left(F\left(\alpha_{1}\right)\right)$ is actually first order definable over $F\left(\alpha_{3}\right)$, and hence there is an $\alpha_{4}<\omega_{y}$ such that $\mathfrak{F}\left(F\left(\alpha_{1}\right)\right)$ equals $F\left(\alpha_{4}\right)$. This gives the induction from $\alpha$ to $\alpha+1$. Similar arguments will take care of the induction at limit ordinals. $\mathrm{M}_{\omega_{y}} \subseteq$ $F\left(\omega_{\nu}\right)$ then follows by induction and the nature of the function $F$. The converse is easy. (Received 55 July, I965.)

Joan Rand Moschovakis. Disjunction and existence in formalized intuitionistic analysis.

For the common portion of intuitionistic and classical analysis, as formalized by Kleene in S. C. Kleene and R. E. Vesley, Foundations of intuitionistic mathematics, Amsterdam (North Holland) 1965, we obtain the following.

Theorem. For closed formulae A, B, $\exists x \mathrm{~A}(\mathrm{x})$ : 
i) $\vdash \mathrm{A} \vee \mathrm{B}$ only if $\vdash \mathrm{A}$ or $\vdash \mathrm{B}$,

ii) $\vdash \mathrm{GxA}(\mathrm{x})$ only if $\vdash \mathrm{A}(\boldsymbol{x})$ for some numeral $\boldsymbol{x}$.

This extends results of Gödel, Gentzen and Kleene for intuitionistic propositional calculus, intuitionistic predicate calculus and intuitionistic number theory.

The theorem also holds for numerous weaker subsystems of intuitionistic analysis. (Received I5 July, I965.)

J. M. B. Moss. Syntactic and semantic paradoxes.

1. Bennett has recently suggested (this Journal 30, 101-2) that any puzzle concerning the prediction paradox requires for its formulation epistemological rather than deducibility concepts. Cargile (this Journal 30, 102-3), however, obtains a deducibility interpretation of the announcement but as a liar-type semantic paradox. In opposition to these and to others who have written on the problem, the first half of the present paper gives a reformulation of the announcement

$C$ : There will be a surprise test one day next week

which (i) can be true

(ii) uses a deducibility notion

(iii) uses modal logic

(iv) applies to any number $n$ of days $(n \geqq 1)$.

The reformulation is as follows. $C=A^{\prime} \& B^{\prime}$ where

$A^{\prime}:$ A test occurs on one of the days $\mathrm{d}_{1}, \ldots, \mathrm{d}_{n}(n \geqq 1)$

$B^{\prime}$ : For each $i \leqq n$, it cannot be deduced from $A^{\prime}, B^{\prime}$, and from the non-occurrence of the test before $d_{i}$, whether or not the test occurs on $d_{i}$.

For simplicity only the case $n=1$ is considered, for which $C=A \& B$. $A$ is $A^{\prime}$ for $n=1$, and $B^{\prime}$ simplifies to

$B$ : It cannot be deduced from $A \& B$ whether or not $A$ is true.

(Trivially $A \& B \vdash A$ but it does not follow that whether or not $A$ holds can be deduced from $A \& B$. For $A \& B$ might not be provably consistent in which case $A \& B \vdash-A$ cannot be eliminated.) The following formulation of $D$ (it can be deduced from $P$ whether or not $Q)$ is suggested.

$D:(\vdash \diamond \mathrm{P}) \&((\vdash \mathrm{P} \supset \mathrm{Q}) \vee(1-\mathrm{P} \supset-\mathrm{Q}))$.

For the present argument, deducibility is for the system of modal (S5) propositional logic with ' $F$ ' as an additional logical symbol, in which $A$ ', $B^{\prime}, A$ can be expressed, and with the additional axiom schemata

(i) $\diamond \mathrm{P}$ where $\mathrm{P}$ is contingent,

(ii) $(\diamond \vdash \mathrm{P}) \supset \vdash \mathrm{P}$.

We have the following formalized version of $B$.

$B:(ー \vdash \diamond(A \& B)) \vee-((\vdash A \& B \supset A) \vee(\vdash A \& B \supset-A))$

whence

$\diamond C \equiv-\vdash \diamond C$

Thus $C$ is possibly true, although this is not provable. $C$ is true iff $A$ is true. Also $B$ is true and unprovable. This solves the 'paradox'.

2. $B$ is syntactically self-referential. Although by standard methods (e.g. Fitch in A.P.Q. 1, 161-4) in many formal systems sentences can be found which are true iff unprovable, no such Gödelian sentence is either genuinely self-referential or synonymous with $B$. Nor is it necessary that anyone who understands $B$ or $C$ be familiar with the method of arithmetization.

The above formalized version of $B$ does however blur the distinction between object and metalanguage. This leads to no difficulties and $B$ is no more obscure than

$E$ : This sentence contains five words.

Although syntactic self-reference is unproblematic, semantic self-reference is sometimes paradoxical and always puzzling. The puzzling features are analogous to those 
that arise with impredicatively defined sets, and it is unfortunate that this analogy, recognised by Russell and Poincare in the first decade of the century, has since been disregarded. The recently developed cumulative type theory can, however, be related to Tarski's language hierarchy. Alternatively, semantic concepts can be associated with classes in the NBG sense; for example, the property of heterologicality has logical characteristics similar to those of a proper class. (Received 20 August, 1965.)

GERT MÜLLER. On forms of recursion.

We consider the following scheme for the introduction of functions in the framework of quantifier-free elementary number theory $Z_{0}$ (including some of the usual simple primitive recursive functions) :

$$
\Sigma\left\{\begin{array}{l}
f\left(c_{1}, \ldots, c_{r}, a_{1}, \ldots, a_{p}\right)=g_{0}\left(c_{1}, \ldots, c_{r}, a_{1}, \ldots, a_{p}\right) \text { if } \Pi c_{j}=0 \\
f\left(c_{1}^{\prime}, \ldots, c_{r}^{\prime}, a_{1}, \ldots, a_{p}\right)=T\left[c_{1}, \ldots, a_{p}, f, g_{1}, \ldots, g_{q}\right]
\end{array}\right.
$$

where $f$ is a function sign not used earlier, the $g_{1}(0 \leqq i \leqq q)$ are previously introduced functions and $\mathrm{T}[\ldots]$ is any term syntactically built up from the variables indicated and function signs. (Evidently $\Sigma$ is too strong since it contradicts $Z_{0}$.) Let $\Sigma(\mathrm{T})$ be the scheme which arises from $\Sigma$ by fixing T. - It is shown that

(i) there is a simple syntactical condition $C$ on $T$ such that $(T)$ contradicts $Z_{0}$ iff $\mathrm{C}$ is fulfilled,

(ii) if not-C is fulfilled, $\Sigma(\mathrm{T})$ generates computable functions only,

(iii) $\mathrm{T}$ can be given a simple normal form and

(iv) (using transfinite induction up to $\omega^{\omega}$ ) $\Sigma(\mathrm{T})$ generates exactly the $r$-recursive functions in R. Peter's sense.

Thus in a certain sense the $r$-recursive functions are the most general functions we can get from schemes of the form $\Sigma$. (Received 27 August, 1965.)

\section{G. SACKs. Metarecursion theory.}

Metarecursion theory is a generalisation of recursion theory in which the natural numbers are replaced by the recursive ordinals. Metarecursively enumerable sets of recursive ordinals are defined with the help of Kleene's theory of notations for recursive ordinals and $\Pi_{1}$ sets. The bounded, metarecursive sets behave like the finite sets of recursion theory and, for that reason, are called metafinite. An equation calculus analogous to Kleene's and devised by Kripke is used to generalise various results of recursion theory such as the solution to Post's problem and the maximal set construction. The metarecursively enumerable sets of finite ordinals coincide with the $\Pi_{1}^{1}$ sets of natural numbers. This makes it possible to apply priority methods to $\Pi_{1}^{1}$ sets, and it can be shown there exists a maximal $\Pi_{1}^{1}$ set. A paper on this subject, by Kreisel and Sacks, will appear shortly in this JOURNAL. (Received 26 July, I965.)

\section{G. STAHL. The effectivity of questions.}

Following the articles of the author: Un developpement de la logique des questions, Revue philosophique de la France et de l'etranger, Paris 1963, 293-301, and Preguntas y premisas, Revista de Filosofia, Santiago, 1961, No. 1, 3-9, questions are introduced as classes of certain wellformed expressions, which are called "sufficient answers", so that the relation between a question and its (sufficient) answers is that between a class and its elements. These questions may be treated with respect to different systems, a fact which is important, because in certain cases theorems are included in the sufficient answers while the negations of theorems are always excluded.

Now the paper gives in several steps a demonstration that questions as to decidable systems are effective or at least effectively enumerable, but that they are not even effectively enumerable as to undecidable systems. It seems excessive to demand effectivity for questions but effective enumerability is a highly desirable condition; 
for the effective enumerability means that if someone presents a supposed (sufficient) answer to a question he has to justify his presentation, and we will be able to check if the justification is really a justification or not.

In order to obtain effective enumerability for questions as to undecidable systems, two alternative definitions of "question" are presented, each of which sacrifices, in a certain measure, a very important point, the exclusion of the negations of theorems. (Received I5 June, I965.)

ROGER F. WheELER. Complete connectives for the 3-valued propositional calculus.

The paper gives necessary and sufficient conditions for a propositional function of $n$ arguments in 3-valued logic to be a complete connective. Previous sets of known necessary conditions had only been proved to be sufficient for the case of binary functions. The present set of conditions enables an explicit formula for the number of complete connectives of $n$ arguments in the 3-valued propositional calculus to be derived. (Received 29 June, 1965.)

F. RivetTi Barbó. Richard's paradox: its root and solution.

Richard's paradox: to each definition of numbers (with a finite number of words) a natural number is associated thus forming an enumerable set $\mathrm{E}$. By Cantor's method a non-enumerable number $\mathrm{N}$ is defined by using a finite phrase $\mathrm{G} ; \mathrm{G}$ being enumerable, we have the paradox.

Richard avoids it by excluding impredicative definitions like $\mathrm{G}$.

I advocate a simpler and less onerous solution, which depends on the very root of the formulability of the paradox, if considered from the logico-philosophical standpoint.

The non-enumerability of $\mathrm{N}$ being proved, this does not involve anything about the enumerability of $\mathrm{G}$; although the non-enumerability of $\mathrm{N}$ depends on the definition $\mathrm{G}$ itself. The paradox would hold if and only if $\mathrm{N}$ would have the same properties as the number associated with $\mathrm{G}$ : but this is not proved.

The property of $G$ (being a finite phrase) on which its enumerability relies, is independent from the properties of its meaning and of the object defined by $\mathrm{G}$ (this may be infinite, non-enumerable, etc.).

Denying this independence involves the impossibility of mentioning anything infinite (be it enumerable or not), as names and definitions have a finite number of signs.

The reason is that one object may be viewed through many aspects; thus the number $\mathrm{N}$ is designated by means of $\mathrm{G}$, and also by a numeral (described as having infinite decimals). $G$ denotes $N$ through its sense, this sense describing a numeral by its relations to other numerals; and the numeral so described denotes $\mathrm{N}$ through its own sense. (Received I7 July, 1965.)

G. Beaman. The modality of deductive systems.

Let $\mathrm{S}$ be a set of propositions closed under Boolean operations and let $\mathrm{V}$ be a proper non-empty subset of $\mathrm{S}$ which contains both $\mathrm{P}$ and $\mathrm{Q}$ iff it contains the conjunction $\mathrm{P}$.Q. Then $\mathrm{V}$ is a filter of $\mathrm{S}$ and $(\mathrm{S}, \mathrm{V})$ is a deductive system.

If $\mathrm{P} \varepsilon \mathrm{V}$ we call $\mathrm{P}$ valid in $(\mathrm{S}, \mathrm{V})$. If $\mathrm{W}$ is a filter such that the system $(\mathrm{S}, \mathrm{V})$ is stronger than (S, V).

If $\neg \mathrm{P} \& \mathrm{~V}$ we say $\mathrm{P}$ is consistent with $\mathrm{V}$. This is because there then exists a stronger system than $(S, V)$ in which $P$ is valid.

The problem we treat is that of embedding $(\mathrm{S}, \mathrm{V})$ in a system $\left(\mathrm{S}_{\mathrm{m}}, \mathrm{V}_{\mathrm{m}}\right)$ with modality in such a way that $\square$ and $\diamond$ (defined as $\square \neg$ ) can be read as expressing validity and consistency respectively. Precisely, $S$ is to be a Boolean subalgebra of $S_{m}$, and

$$
\mathrm{V}=\mathrm{S} \cap \mathrm{V}_{\mathrm{m}} \text {. }
$$


Since $\square \mathrm{P}$ is to be read as expressing the validity of $\mathrm{P}$ in $(\mathrm{S}, \mathrm{V})$ we have

$$
\mathrm{P} \varepsilon \mathrm{V} \rightarrow \mathrm{P} \varepsilon \mathrm{V}_{\mathrm{m}}
$$

Since $P . Q$ is valid iff both $P$ and $Q$ are, the equality

is to hold in $S_{m}$.

$$
\square(P . Q)=\square P \cdot \square Q
$$

If a proposition is valid in a system, then it is valid in a stronger system. But consistency is not similarly conserved. A proposition which is consistent in the weaker system may be false in a stronger one. Consider then the interpretation of $\square \diamond P$. To say that $\diamond \mathrm{P}$ is valid is to say that $\mathrm{P}$ has a permanent consistency, that $\mathrm{P}$ cannot be made invalid by strengthening the system. But this is so if and only if $\mathrm{P}$ is valid. Thus we have an identification of modalities

$$
\square \diamond \mathrm{P}=\square \mathrm{P}
$$

The postulates (3) and (4) define $S_{m}$, the postulates (1) and (2) define $V_{m}$.

Simplifying $\square \diamond \square \mathrm{P}$ in two ways gives the equation

$$
\square \square \mathbf{P}=\square \mathbf{P} \text {. }
$$

It may be shown easily that removing any modal operator which stands in the scope of a modal operator gives a proposition equal to the original one.

Discussion of the topic with Dawson and Prior has been of great value. (Received 5 July, 1965.)

J. P. CleAve and H. E. Rose. $\mathscr{E}^{\mathbf{n}}$-Arithmetic.

$\mathscr{E}^{\mathrm{n}}$ is the class of recursive functions, defined in [1], having the successor function, $\mathrm{u}_{1}(\mathrm{x}, \mathrm{y})=\mathrm{x}, \mathrm{u}_{2}(\mathrm{x}, \mathrm{y})=\mathrm{y}$ and $\mathrm{f}_{\mathrm{n}}(\mathrm{x}, \mathrm{y})$, given by

$$
\begin{aligned}
\mathbf{f}_{\mathbf{0}}(x, y) & =y+1 \\
f_{1}(x, y) & =x+y \\
f_{2}(x, y) & =(x+1)(y+1) \\
t_{n+3}(0, y) & =f_{n+2}(y+1, y+1) \\
f_{n+3}(x+1, y) & =f_{n+3}\left(x, f_{n+3}(x, y)\right),
\end{aligned}
$$

as initial functions and is closed under substitution and limited recursion.

Theorem 1. $\mathscr{E}^{n}=\mathscr{E}^{n}$, where the class of recursive functions $\mathscr{E}^{\mathrm{n}}$ is defined as above except that $f_{n}(x, y)$ is replaced by the Ackermann function $g_{n}(x, y)$ defined by

(Solution of problem 7 in [1].)

$$
\begin{aligned}
g_{0}(x, y) & =y+1 \\
g_{1}(x, y) & =x+y \\
g_{2}(x, y) & =x \cdot y \\
g_{n+3}(0, y) & =1 \\
g_{n+3}(x+1, y) & =g_{n+2}\left(g_{n+3}(x, y), y\right)
\end{aligned}
$$

$\mathscr{E}^{\mathrm{n}}$-arithmetic is the free variable system of arithmetic obtained from that defined in [2] by replacing recursively defined functions by the functions in $\mathscr{E}^{\text {n }}$. $\vdash_{\text {n }}$ denotes derivability in $\mathscr{E}^{\mathrm{n}}$-arithmetic. Let $\mathrm{H}(\mathrm{x})$ denote a formula of $\mathscr{E}^{\mathrm{n}}$-arithmetic with free

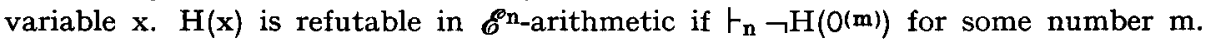
$\mathrm{T}_{\mathrm{n}}$ and $\mathrm{R}_{\mathrm{n}}$ denote the sets of provable and refutable formulae, respectively, of $\mathscr{E}^{\mathrm{n}}$ arithmetic.

Theorem 2. For $\mathrm{n} \geqq 2, T_{n}$ and $R_{n}$ are effectively inseparable.

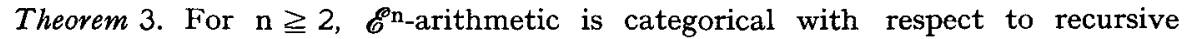
models (cf. [3]).

Theorem 4. For $\mathrm{n} \geqq 2, \vdash_{\mathrm{n}+1} \operatorname{Consis}\left(\mathscr{E}^{\mathrm{n}}\right.$-arithmetic).

[1] A. Grzegorczyk, Rozprowy Mat. (IV), 1953.

[2] H. E. Rose, Zeit. f. Math. Logik (7) 1961.

[3] G. Kreisel, J.S.L. (23) 1958. (Received ro Sept., 1965.) 
Jens ERrk Fenstad. A short proof of Craig's interpolation theorem.

Using basic facts about ultraproducts and elementary topological properties of the spaces of models we are able to give a short proof of Craig's interpolation theorem:

Let $\varphi$ and $\psi$ be sentences having common relational symbols and assume that $\vdash \varphi \rightarrow \psi$. Let $\mathrm{R}_{\mathbf{1}}$ be the similarity type of $\varphi$ and $\psi$ and define $\mathrm{K}_{\mathbf{1}}=\left\{\mathfrak{U} \varepsilon \mathrm{R}_{\mathbf{1}}: \mathfrak{A} \models \varphi\right\}$, $\mathrm{K}_{2}=\left\{\mathfrak{A} \varepsilon \mathrm{R}_{1}: \mathfrak{A} \models \neg \psi\right\}$. Denote by $\mathrm{R}_{0}$ the similarity type of the common vocabulary of $\varphi$ and $\psi$, and let red be the reduction map $\mathbf{R}_{\mathbf{1}} \rightarrow \mathbf{R}_{\mathbf{0}}$. A simple but useful fact is that $r e d$ is continuous in the topologies induced by $\mathrm{EC}$ sets. Let $\mathrm{R}_{\mathbf{0}}=\mathrm{R}_{\mathbf{0}} / \equiv$ (considered in the quotient topology). Further let $N_{1}=\operatorname{red}\left(\mathrm{K}_{1}\right)$ and $\mathrm{N}_{i}^{\prime}=\pi\left(\mathrm{N}_{\mathrm{i}}\right)$, where $\pi: \mathrm{R}_{0} \rightarrow$ $R_{6}{ }^{\prime}$ is the projection map. As $R_{0}^{\prime}$ is Hausdorff, $N_{i}^{\prime}$ is closed.

Lemma. $N_{1}^{\prime} \cap N_{2}^{\prime}=\varnothing$. - If not, there are models $\mathfrak{A}_{1} \varepsilon \mathrm{N}_{1}$ such that $\mathfrak{A}_{1} \equiv \mathfrak{A}_{2}$. By a theorem of Kochen elementary equivalent systems have isomorphic ultralimits, and it is easy to verify that the pseudo-elementary classes $N_{1}$ are closed under ultralimits. Thus one may construct an $\mathfrak{A} \varepsilon \mathrm{K}_{1} \cap \mathrm{K}_{2}$, contradicting $\vdash \varphi \rightarrow \psi$.

Hence $\mathrm{N}_{1}^{\prime}$ and $\mathrm{N}_{2}^{\prime}$ can be separated by an open set in $\mathrm{R}_{0}$, which means, going back to $R_{0}$, that there is an open set $N_{0}$ such that $N_{1} \subseteq N_{0}$ and $N_{0} \cap N_{2}=\varnothing$. A compactness argument then gives an elementary class $N_{\theta}$ separating $N_{1}$ and $N_{2}$, which implies that there is a sentence $\theta$ in the common vocabulary of $\varphi$ and $\psi$ such that $\vdash \varphi \rightarrow \theta$ and $\vdash \theta \rightarrow \psi$. (Received Io Sept., 1965.)

Matthew J. Hassetr. A closure property of regressive isols.

Let $\Lambda_{R}$ denote the collection of all regressive isols. J. Barback has shown that the canonical extension of a recursive function of one variable maps $\Lambda_{R}$ into $\Lambda_{R}$ if and only if that function is eventually increasing (Math. Scand. 15 (1964), 29-42). The following answers the corresponding question for recursive functions of two variables.

Let $f(x, y)$ be a recursive function of two variables. $f$ will be called flat if there exists a recursive function $g(x, y)$ such that (i) $(\forall x)(\forall y)[f(x, y)=\Sigma \quad \Sigma g(i, j)]$ and (ii) $(\exists \mathrm{n})(\mathrm{Gk})[\mathrm{x}>\mathrm{n} \vee \mathrm{y}>\mathrm{k} \rightarrow \mathrm{g}(\mathrm{x}, \mathrm{y})=0]$. $\mathrm{f}$ will be called reducible to the case of $a$ single variable if (i) there exist eventually increasing recursive functions $\mathrm{f}_{\mathrm{i}}(\mathrm{y}), \mathrm{i}=$ $0, \ldots, m$ such that for $x \leqq m, f(x, y)=f_{x}(y)$ and for $x>m, f(x, y)=f_{m}(y)$ or (ii) condition (i) holds with the roles of $x$ and $y$ interchanged.

Theorem. The canonical extension of $f(x, y)$ maps $\Lambda_{R} \times \Lambda_{R}$ into $\Lambda_{R}$ if and only if there exists an integer $\mathrm{n}$ such that:

(1) For $i<n, f(i, y)$ is an eventually increasing function of $y$ and $f(x, i)$ is an eventually increasing function of $x$, and

(2) $f(x+n, y+n)=m(x, y)+\left(c_{1}(x, y)-c_{2}(x, y)\right)$, where $c_{1}$ and $c_{2}$ are flat recursive functions and $m(x, y)$ is either:

(i) reducible to the case of a single variable or;

(ii) of the form $\min (\mathrm{g}(\mathrm{x}), \mathrm{h}(\mathrm{y}))$, where $\mathrm{g}(\mathrm{x})$ and $\mathrm{h}(\mathrm{y})$ are eventually increasing recursive functions of one variable.

Corollary. The canonical extension of the maximum function does not map $\Lambda_{\mathbf{R}} \times \Lambda_{\mathbf{R}}$ into $\Lambda_{\mathbf{R}}$.

This corollary is of particular interest in view of the previously established fact that the canonical extension of the minimum function does map $\Lambda_{R} \times \Lambda_{R}$ into $\Lambda_{R}$. (Received I5 July, 1965.)

A. H. Lachlan. Three theorems about r.e. sets.

Theorem 1. If $\alpha$ is hyperhypersimple but not maximal, then there exist complementary r.e. sets $\beta_{1}, \beta_{2}$ such that $\alpha=\beta_{1} \cap \beta_{2}$.

Theorem 2. Let $\alpha$ be any non-recursive r.e. set, then there exists an r.e. subset $\beta$ of $\alpha$ such that $\alpha-\beta$ is infinite and such that if $\delta$ is any r.e. complement of $\alpha$ then $\beta \cup \delta$ is cofinite. 
For the definition of covering number of an r.e. set see Mathematical Reviews 26 (1963), p. 691.

Theorem 3. There is a hyperhypersimple set with no maximal superset and which has covering number $\infty$.

Using Theorem 1 the r.e. sets with finite covering numbers can be characterised as those discovered by Yates (Z. Math. Logik 8 (1962), 344). From Theorems 1 and 2 we obtain another proof of Martin's result that there exists an r.e. set with no hyperhypersimple superset. (Received I5 July, I965.)

TRENChARD MORE, Jr. Computer generation of truth-tables for implicational calculi.

Each unlabeled node in the lattice of implicational calculi on page 160 , vol. 29 of this Journal merges with the labeled node that covers it. Entailment plus modified Pierce gives modified iteration. Entailment plus half-modified iteration plus classical Peirce gives classical iteration. This last result suggested and was obtained from Professor Fitch's privately communicated proof that S5 plus classical Peirce gives the classical two-valued logic.

A computer program, which uses to advantage the table look-up and variable field length features of the IBM 1620 , has generated all (to within isomorphism and having no proper homomorphic images) regular (if $\mathrm{A} \Rightarrow \mathrm{B}$ and $\mathrm{A}$ are designated, so is $\mathrm{B}$ ) 3 by 3 and 4 by 4 truth tables that satisfy every provable formula of pure entailment. The program classified the truth-tables as models for each of the nine calculi corresponding to the labeled nodes of the lattice mentioned above. (Received $15 \mathrm{July}$, rg65.)

Albert A. Mullin. On the possibility of applications of modern logic to physical problems.

It is well known from the works of C. Peirce, J. von Neumann, G. Birkhoff, C. Shannon, P. Suppes and many others that logic has applications to physical theory; e.g., whereas classical mechanics has a Boolean algebra as its underlying logic, classical quantum theory has a more general lattice-theoretical foundation. The present physico-metamathematical and philosophical inquiry discusses, among other matters, use of analogies with elementary ideas on recursive sets for the theory of null measurements, e.g., with two or more inseparably related compartments, viz., those manifesting themselves through interactions between the measured and the measuring. Two basic problems, starting with initial conditions as "axioms" and physical laws as "rules of inference" are the following: (1) to determine, if possible, the first instant of time that a measured substance is absent and remains absent from its compartment and (2) to determine, if possible, for a particle with prescribed momentum which compartment it is in. The first problem reduces to the decision problem as to whether a compartment is, in fact, empty or merely contains substance that has not yet been activated by the measuring instrument. The second decision problem, subject to the limitations of the Uncertainty Principle, is closely related to the problem of "Maxwell's Demon", and hence to the impossibility of Perpetuum Mobile of the second kind.

Finally, the author presents arguments to defend the theses that (i) none of the three "laws of thought" entail the necessity (suitably defined for recursively axiomatizable systems) of physical events (use is made of Sir W. Hamilton's "Axe" relating to the quantification of predicates) and (ii) independence statements such as the "parallel postulate" and the "continuum hypothesis" can 'say' something about physical reality rather than just serving as the bases for non-Euclidean geometries and non-Cantorian set theories. (Received 15 July, 1965.)

C. E. M. YATES. Density and incomparability in the degrees less than $\mathbf{0}^{(1)}$.

Sacks has proved that the recursively enumerable degrees are dense (Ann. of Math., 1964), in other words, between any two comparable recursively enumerable 
degrees there is another recursively enumerable degree. It is natural to ask to what extent one can interpolate recursively enumerable degrees between comparable degrees which are merely supposed to be $\leq \mathbf{0}(1)$. The existence of a minimal degree $<\mathbf{O}^{(1)}$ (shown by Sacks) shows that some condition is necessary. The following theorem shows that in general both the given comparable degrees need to be recursively enumerable. Theorem $I$. If $\mathbf{a}$ is any non-zero recursively enumerable degree (in particular if $\mathbf{a}=\mathbf{0}(1)$ ) then there is a non-zero degree $\mathbf{b}<\mathbf{a}$ which is not comparable with any non-zero recursively enumerable degree $<\mathbf{a}$. Next we prove: Theorem 2 . If a and $\mathbf{b}$ are non-zero recursively enumerable degrees such that $\mathbf{a}<\mathbf{b}$ then there is a degree $\mathbf{c}$ such that $\mathbf{a} \cup \mathbf{c}=\mathbf{b}$. (By a theorem of Lachlan the degree $\mathbf{c}$ in theorem 2 cannot always be chosen to be recursively enumerable.) Lastly, we answer a question of Sacks (Degrees of unsolvability, (Annals of Mathematics Studies, 55, 1963)) by showing Theorem 3 there is a degree between $\mathbf{0}$ and $\mathbf{0}(1)$ with no minimal degree less than or equal to it. (Received I5 July, 1965.)

There will be a meeting of the Association for Symbolic Logic at the Waldorf-Astoria Hotel in New York on Monday, April 4, 1966, in conjunction with a meeting of the American Mathematical Society. Members wishing to contribute papers will please submit abstracts of not over 300 words in duplicate before February 15, 1966 sending them to Professor Martin Davis, Department of Mathematics, New York University, University Heights, New York, New York 10453.

On April 5, 6, and 7, there will be a Symposium on Mathematical Aspects of Computer Science jointly sponsored by the Association for Computing Machinery, the Association for Symbolic Logic and the American Mathematical Society, and supported financially by the Air Force Office of Scientific Research, the Institute for Defense Analyses, and the U.S. Army Research Office - Durham.

\section{SUMMER MEETING IN HANNOVER, GERMANY}

There will be a Colloquium on Logic and Foundations of Mathematics at Hannover, Germany, from August 8 to 12,1966, organized by the DVMLG (Deutsche Vereinigung für Mathematische Logik und für Grundlagenforschung der exakten Wissenschaften). It will be held before the Moscow-International Congress so that persons intending to go to Moscow will be able to attend. This Colloquium has the sponsorship of the Association for Symbolic Logic as its 1966 European meeting and, in favor of this Colloquium, there will be no logic Colloquium in England for 1966.

The Annual Meeting of the Association for Symbolic Logic will be held in Houston, Texas, on January 23 and 24, 1967. It will be in conjunction with the Annual Meeting of the American Mathematical Society (January 24 - January 27) and a meeting of the Mathematical Assocation of America (January 27-28). Members wishing to submit papers are requested to send abstracts (not exceeding 300 words in length) in duplicate to J. A. Robinson, Rice University, Houston, Texas, or to Norman M. Martin, University of Texas, Austin, Texas, by November 10, 1966. 